\title{
Electrostatic Tuning of the Properties of Disordered Indium Oxide Films near the Superconductor-Insulator Transition
}

\author{
Yeonbae Lee, ${ }^{1}$ Aviad Frydman, ${ }^{2}$ Tianran Chen,${ }^{1}$ Brian Skinner,${ }^{1}$ and A. M. Goldman ${ }^{1}$ \\ ${ }^{1}$ School of Physics and Astronomy, University of Minnesota, \\ 116 Church St. SE, Minneapolis, MN 55455, USA \\ ${ }^{2}$ Department of Physics, Bar Ilan University, Ramat Gan 52900, Israel
}

\begin{abstract}
The evolution with carrier concentration of the electrical properties of amorphous indium oxide $\left(\mathrm{InO}_{x}\right)$ thin films has been studied using electronic double layer transistor configurations. Carrier variations of up to $7 \times 10^{14} \mathrm{carriers}_{\mathrm{c}} \mathrm{cm}^{2}$ were achieved using an ionic liquid as a gate dielectric. The superconductor-insulator transition was traversed and the magnitude and position of the large magnetoresistance peak found in the insulating regime were modified. The systematic variation of the magnetoresistance peak with charge concentration was found to be qualitatively consistent with a simulation based on a model involving granularity.
\end{abstract}

The competition between disorder and superconductivity has long been the subject of theoretical and experimental study [1 6]. By varying the nominal disorder a superconductor-insulator transition (SIT), known as disorder-driven SIT, is found in materials such as amorphous $\mathrm{Bi}(a-\mathrm{Bi})[7]$, TiNx [8], and $\mathrm{InOx}[9$, 10]. Such transitions in disordered thin films are believed to be quantum phase transitions. Among the materials, amorphous $\mathrm{InO}_{x}$ (which we will refer to as $\mathrm{InO}$ ) is of great interest because of the novel feature of a giant magnetoresistance (MR) peak at low temperatures [10 13]. Moreover direct evidence of Cooper pairs, both above the transition temperature, and in the insulating regime has been reported 14, 15]. However, the study of the disorderdriven SIT in InO, mainly achieved by varying its oxygen concentration during growth and heat treatment afterward, can introduce unavoidable complexities to the system including the conflation of variations of carrier concentration and levels of disorder 12, 16].

In this Letter, we overcome these limitations by using a different approach. Namely, we tune the carrier concentration by adapting a field effect transistor (FET) configuration, and we use this method to induce a SIT within a given sample without altering its disorder (In earlier work, Parendo et al 17 reported the study of the electrostatically tuned SIT of $a$-Bi.). Benefiting from the FET configuration, we present a comprehensive study of the SIT driven by carrier modulation. This has revealed many interesting phenomena such as variable range hopping in the insulating regime and the broadening of superconducting fluctuations near the SIT. This new approach also enables us to produce and then continuously change the size and the position of MR peak in the insulating regime. We believe that our findings shed light on the origin of the MR peak and the possibility of localized superconducting islands within the insulating regime.

Amorphous (meaning non-crysal within our context) indium oxide ( $\mathrm{InOx}$ ) films were e-gun evaporated using $99.999 \%$ pure $\mathrm{In}_{2} \mathrm{O}_{3}$ pallets. The films were grown on
Si wafers (100) through a shadow mask defining a Hall bar geometry. The base pressure in the deposition chamber was $2 \times 10^{-7}$ mbar. Pure $\mathrm{O}_{2}$ gas with partial pressure $4 \times 10^{-6}$ mbar was bled into the chamber during the evaporation in order to achieve samples with sheet resistances of about $10 \mathrm{k} \Omega$ at $T=4 K$, close to the SIT. Sample thicknesses ranged from $10 \mathrm{~nm}$ to $6 \mathrm{~nm}$. The $6 \mathrm{~nm}$ samples were all insulating as grown and are the central focus of this work. Gold electrodes, $50 \mathrm{~nm}$ thick, were deposited on the InO films. An ionic liquid (DEME-TFSI) was used as a gate dielectric with a $\mathrm{Pt}$ coil top gate to form an electric double layer transistor (EDLT). A schematic of the top-gated EDLT can be found in our previous work [18]. (Earlier, Misra, McCarthy, and Hebard gated InO films with ILs but did not explore their superconductivity [19].) The sheet resistances $R_{s}$ of the films were determined employing a four-probe electrode configuration. A ${ }^{3} \mathrm{He}$ refrigerator with a superconducting magnet enabled us to vary the temperature between $300 \mathrm{~K}$ and $0.40 \mathrm{~K}$ and the magnetic field between $0 \mathrm{~T}$ and $9 \mathrm{~T}$. Gate voltages $V_{g}$, ranging from $+3 \mathrm{~V}$ to $-3 \mathrm{~V}$ were used to induce or deplete charge carriers, which are electrons. The various values of $V_{g}$ were applied at a temperature of $240 \mathrm{~K}$ and were held constant throughout the subsequent cooling and measurement.

The $T$ dependencies of $R_{s}$, of two InO samples at different values of $V_{g}$ are plotted in Fig. 1. Both samples were grown under the same conditions i.e., at the same oxygen pressure and at the same time. However their initial resistances were adjusted so as to be different, by annealing at $65^{\circ} \mathrm{C}$ under vacuum for different periods of time. Both samples exhibited large changes in $R_{s}$ upon gating. Unfortunately, Hall effect measurements of such highly disordered systems were not possible, except in the case of a few other samples (not shown) that had relatively low normal state resistances. The process of gating was found to be reversible, with minor hysteresis. This suggests that apart from the remote possibility of a reversible chemical reaction, the gating process is electrostatic. To 

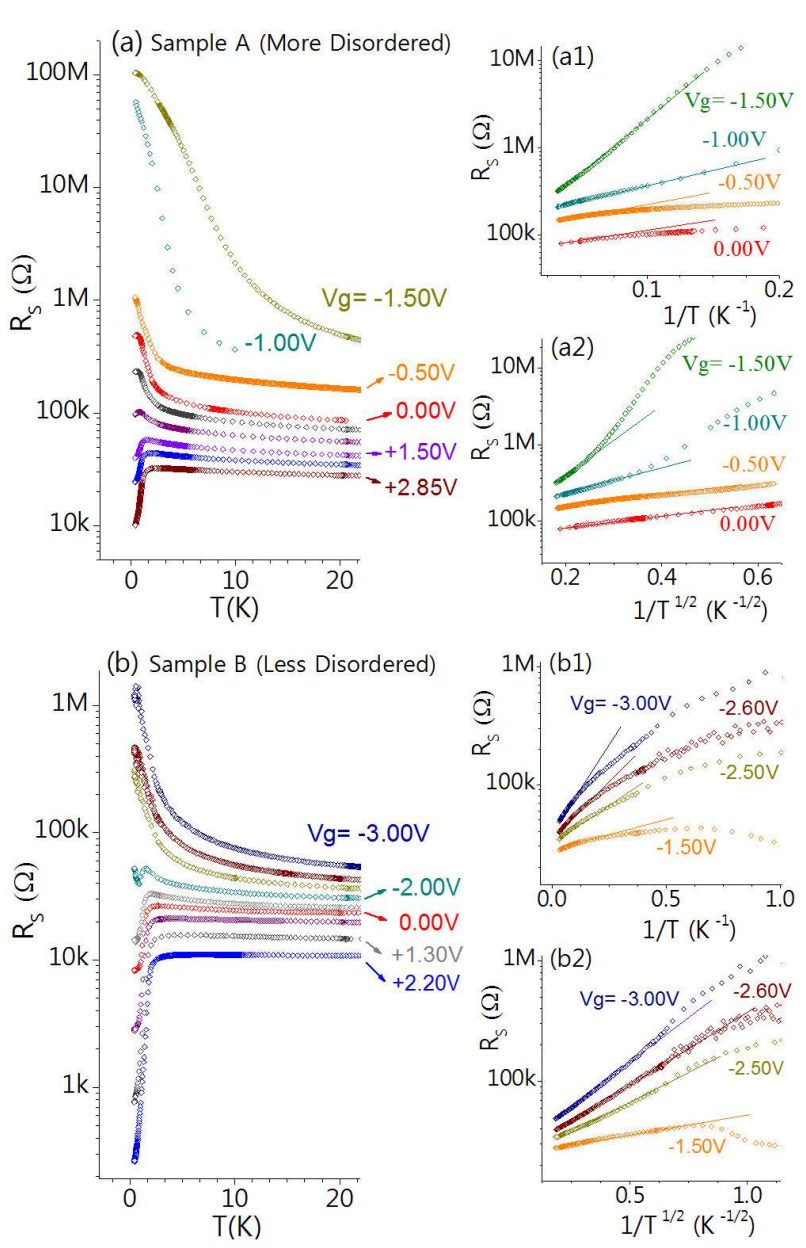

Figure 1: (Color online) The temperature dependence of $R_{s}$ at various values of $V_{g}$ of samples A (a) and B (b). The onset of superconductivity (the initial downward dip in the $R_{s}$ vs. $T$ curve) occurs at $V_{g}=+1.00 \mathrm{~V}$ for sample A, and at $V_{g}=-2.00 \mathrm{~V}$ for sample $\mathrm{B}$ respectively. On the right hand panels, both $R_{s}$ vs. $1 / T$ and $1 / T^{1 / 2}$ are plotted for sample A (a1-a2) and for sample B (b1-b2). Note the cross-over from Arrhenius to ES VRH at $V_{g}=-0.50 \mathrm{~V}$ shown in (a1-a2), whereas (b1-b2) shows ES VRH over all ranges of $V_{g}$.

determine and/or confirm the carrier modulation of the system, we used an electrochemical technique known as the Chronocoulometry[20]. Using this technique, we observed changes in the sheet (2D) carrier density or charge transfer $\left(\Delta n_{\text {sheet }}\right)$ of up to $7 \times 10^{14}$ carriers $/ \mathrm{cm}^{2}$. However we did not characterize the charge transfer at each gate voltage for the films reported here.

The accessible charge transfer was large enough for us to observe the SIT of InO as shown in Figs. 1(a) and 1(b). The right hand panels of Fig. 1 show $R_{s}$ vs. $T$ behavior of the insulating state; both Arrhenius $R=R_{0} \exp \left(T_{0} / T\right)$ and Efros-Shklovskii variable range hopping (ES VRH) $R=R_{0} \exp \left(T_{0} / T\right)^{1 / 2}$ were observed. Comparing Figs. 1(a1) and 1(b1), the higher resistance sample shows a wider range of Arrhenius activated transport, suggesting

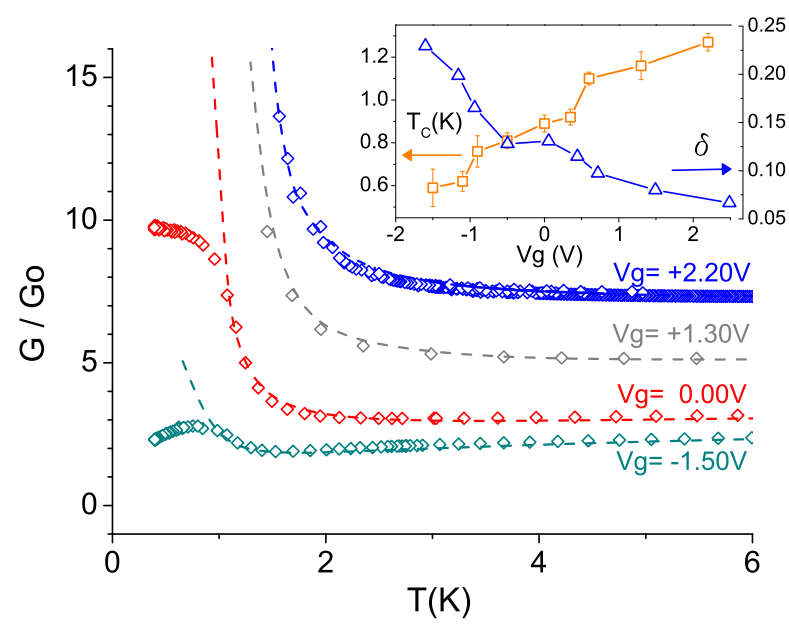

Figure 2: (Color online) Dimensionless conductance $G / G_{0}$ vs. $T$ for the sample B in a superconducting state regime. The dashed lines are the best fits to the data. The inset is a plot of $T_{c}$ (left) and $\delta$ (right) vs. $V_{g}$.

that it has a hard gap in its single-particle density of states. A cross-over from Arrhenius to ES hopping is observed as the film becomes less resistive with increasing carrier concentration. The observed ES hopping suggests the presence of a soft gap (Coulomb gap) in the density of states near the Fermi level, which is due to the long range interactions between electrons in the system[21]. This has also been reported in other systems $[8,22]$. The transition from Arrhenius to ES hopping could be interpreted as the evidence of enhanced electron-electron interaction driven by the addition of carriers. Both samples A and B exhibited SI transitions tuned by carrier modulation, which is clearer in the case of sample B [Fig. 1(b)].

When a system enters a superconducting state below a critical temperature $T_{c}$, global phase-coherence is established with a nonzero amplitude of the superconducting order parameter. Determining $T_{c}$ from data, however, can be a non-trivial task because of the broadening of the superconducting transition due to superconducting fluctuations (SF) above $T_{c}[23]$. Thus, in order to estimate $T_{c}$, we follow a recent theoretical model described in Ref. [24]. According to this model, superconducting fluctuations in $2 \mathrm{D}$ system give rise to a temperature-dependent change in conductance $\Delta G$ given by $\Delta G=\Delta G^{A L+M T}+$ $\Delta G^{D O S}+\Delta G^{W L+E E}$, where $\Delta G^{A L+M T}$ is the combination of the Aslamazov-Larkin (AL) and Maki-Thompson (MT) processes [25, 26], $\Delta G^{D O S}$ accounts for the change in the single-particle density of states (DOS) due to their involvement in fluctuation pairing 24], lastly $\Delta G^{W L+E E}$ is the correction from the weak localization (WL) theory including electron-electron (EE) interactions 27]. With estimated coherence length of $13^{\sim} 20 \mathrm{~nm}$ (Compare this with the typical superconducting coherence length of $10^{\sim} 30 \mathrm{~nm}$ for $\mathrm{InO}[28]$ ), the films with $6 \mathrm{~nm}$ thickness can be treated as (quasi) 2D system enabling us the analysis 
of SF theory in 2D model. To fit the SF fit to the data, we used the asymptotic expression in Table 1 (regime I, the Ginzburg-Landau regime) of Ref. 24]. Figure 2 is the result of the SF fit applied to the data of sample B and the values of both $T_{c}$ and pair breaking parameter $\delta$ are determined simultaneously from the best fit as shown in the inset. This analysis allows us to determine $T_{c}$ and we observe a monotonic increase in $T_{c}$ as function of $V_{g}$. This result implies a monotonic increase of the superconducting pairing energy $\Delta$ as function of carrier density.

The MR measurements of these two samples at different gate voltages are shown in Fig. 3. The most significant feature of the data for sample A [Figs. 3(a1), 3(a2), and $3(\mathrm{a} 3)]$ is the transition from negative to positive MR followed by a downward slope of $R_{s}$ (the MR peak) upon further increase of $H$, all taken at fixed $T$, over the range from $0.5 \mathrm{~K}$ to $1 \mathrm{~K}$. Also note the strong $\mathrm{MR}$ peak found in insulating regimes of both samples. The position of the peak field, $H_{\text {peak }}$, at which the maximum resistance occurs, monotonically increase as function of $V_{g}$.

In a disorder-driven SIT, the spatial inhomogeneity of the pairing energy $\Delta$ can be very important. In the insulating regime, Ghosal et al. [5] suggested that the system breaks up into superconducting islands. Furthermore, in Ref. [6] such a spatial variation of the order parameter amplitude has been demonstrated using a negative U-Hubbard model. Near the SI transition, it has been suggested [29, 30] that the MR peaks in disordered systems arise because magnetic fields affect the concentration and size of superconducting islands, so that as these islands shrink with increasing field, there is a transition from Cooper pair-dominated to single electrondominated transport.

On the other hand, reduction of the superconducting pairing energy $\Delta$ within islands can itself lead to a tradeoff between conduction by Cooper pairs and conduction by unpaired electrons, and thus (potentially) to a MR peak, even when the concentration and the size of the islands are fixed. Recent theoretical works [31, 32] studied a model with fixed size and concentration of superconducting grains (islands), and show how a MR peak deep in the insulating state can arise as a result of the reduction of the superconducting gap $\Delta$ with increased $H$. This predicts that near the MR peak and at low temperature the conduction should be described by ES VRH, as shown in Figs. 1(a2) and 1(b2). Both approaches lead to an insulator in which Cooper pairs with nonzero $\Delta$ are formed in the insulating regime of the system and are responsible for the MR peak.

The shift of the MR peak to higher magnetic fields with increasing carrier concentration, as shown in Fig. 3, can be explained qualitatively within the context of the theory of Ref. [31 33]. Increasing the carrier density presumably increases the density of states at the Fermi level within the superconducting grains, thereby driving up the zero-field superconducting gap, $\Delta_{0}$. A larger $\Delta_{0}$
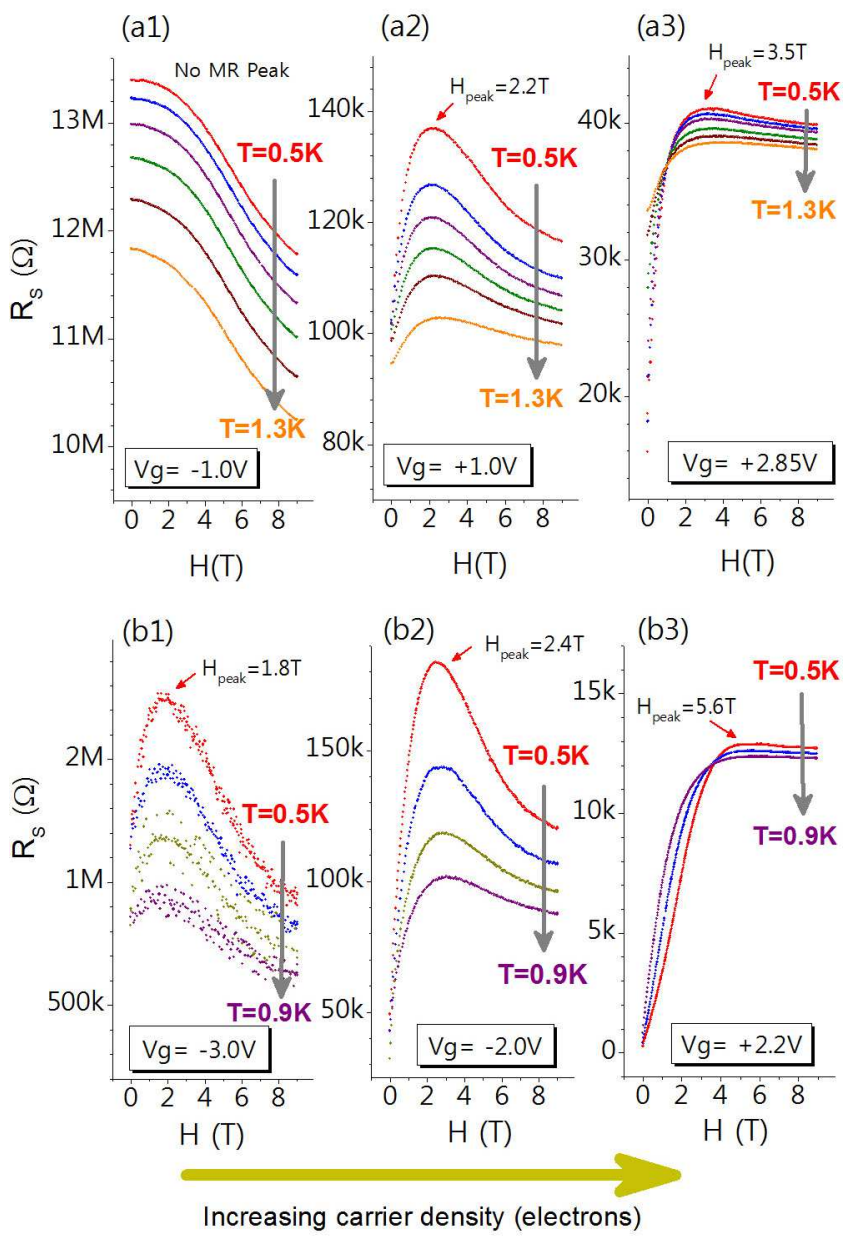

Figure 3: (Color online) $R_{s}$ vs. $H$ at various values of $T$ and $V_{g}$ for sample A, labeled (a1-a3) and sample B, labeled (b1b3). The density of electrons increases from the left to the right. Each curve represents a separate isotherm of $R_{s}$ vs. $H$, where $T$ ranges from $0.5^{\sim} 1.3 \mathrm{~K}$ for sample A (more disordered, top) and from $0.5^{\sim} 0.9 \mathrm{~K}$ for sample B (less disordered, bottom).

implies that a larger $H$ is required in order to reduce $\Delta$ to the value of the grain charging energy $E_{c}$, so that the MR peak shifts to higher $H$. In this way the transition from negative MR [as in Fig. 3(a1)] to a peak at an intermediate $H$ [Fig. 3(a2)] to a peak at a larger $H$ [Fig. $3(\mathrm{a} 3)]$ can be understood.

As an example, Fig. 4 shows values of the resistance of a simulated 2D array of regularly-spaced, monodispersed superconducting grains as a function of $H$, calculated using the method described in Ref. [32]. At small $\Delta_{0} / E_{c}$, the conductivity is primarily due to hopping of unpaired electrons, and there is a monotonic negative MR [as seen, for example, in Fig. 3(a1)]. At larger $\Delta_{0} / E_{c}$, which ostensibly corresponds to larger carrier density, the MR develops a peak associated with a trade-off between conductivity by single electrons and conductivity by Cooper pairs. This peak moves to larger $H$ as $\Delta_{0} / E_{c}$ is increased 


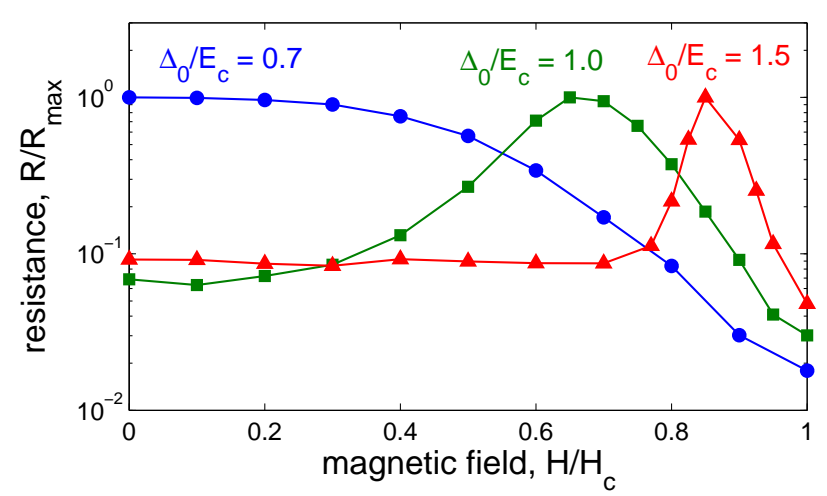

Figure 4: (Color online) Simulation of the resistance of a 2D array of identical superconducting grains deep in the insulating state as a function of $H$. Different curves are labeled with their corresponding values of $\Delta_{0} / E_{c}$, and are normalized to their maximum resistance, $R_{\max }$. As $\Delta_{0}$ is increased, which presumably corresponds to a larger carrier density, a MR peak develops that shifts to larger magnetic field, in qualitative agreement with what is seen in Fig. 3. Here all curves correspond to a temperature such that $k_{B} T=0.1 E_{c}$ and have localization lengths $\xi_{1}$ and $\xi_{2}$ for single-electron and pair conductivity, respectively, satisfying $\xi_{2} / \xi_{1}=8$.

[as in Fig. 3]. For the simulation of Fig. 4 we have assumed a conventional BCS-like dependence of $\Delta$ on the field $H: \Delta=\Delta_{0} \sqrt{1-\left(H / H_{c}\right)^{2}}$. In this way the data shown in Fig. 3 is consistent with the concept of tuning the local superconducting gap by modulating the carrier density. Such tuning of the gap by carrier density also suggests a straightforward possible explanation of the transition from the insulating state to the superconducting state with increasing carrier density. Presumably, the transition may result from regions with zero $\Delta$ being driven to non-zero $\Delta$, thereby connecting superconducting grains that would otherwise be disconnected. Unfortunately, the simulation used to generate Fig. 4 cannot be used for a quantitative determination of the relationship $\Delta(n)$, since this requires a knowledge of the $H$-dependence of the gap as well as the relative localization lengths $\xi_{1}$ and $\xi_{2}$ for unpaired and paired electron hopping. We also caution that the simulation technique is applicable only for the heavily-insulating limit, and in this sense our comparison between Figs. 3 and 4 is only qualitative. It should also be noted that within this simple model a strong MR peak develops only at a relatively large ratio of the localization lengths, $\xi_{2} / \xi_{1}$. A final caveat is the possibility that other models may give similar results.

There is an apparent connection of granular-like superconducting behavior in an amorphous film to sample morphology. Atomic force microscope (AFM) images of these films shown in Fig. 5 (sample B) exhibited thickness roughness which could be interpreted as an effective granularity. In a recent work, ultra thin amorphous $\mathrm{Bi}$

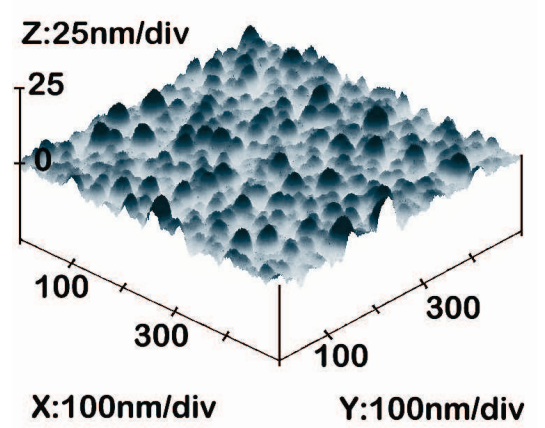

Figure 5: (Color online) The AFM image for sample B revealing strong degree of surface roughness. Similar results were observed for sample A.

films were patterned with nano-scale honeycomb array of holes and MR peaks were observed in several samples with non-uniform film thicknesses [34]. On the other hand the same experiment shows no MR Peaks in samples with uniform film thicknesses 35]. It is also shown that the thickness variations are primarily responsible for producing the localized Cooper pairs in a Bi films [36], and the level of the disorder can be tuned by the variation of the thickness in such systems [7]. Furthermore the study of $\mathrm{InO}$ reported by 13] shows that the different levels of MR anisotropy, which are indirect evidence of the superconducting strength, have a strong relationship to different values of thickness. These findings suggest that there is a close relationship between effective granularity and the localization of Cooper pairs resulting superconducting grains in an insulating matrix. However, a granular morphology may not be essential for a system to exhibit granular like behavior. The oxygen concentration may be the key to determining an effective granularity in the amorphous InO system. A recent experimental work 37] showed that mesoscale spatial variations in the oxygen concentration are present in nominally homogeneous $\mathrm{InO}$ films. Such chemical inhomogeneity can induce local carrier density fluctuations resulting in mesoscale inhomogeneity of the pairing energy $\Delta$. It has also been demonstrated that, by STM measurement, such inhomogeneity can be present even when the sample surface shows high-level smoothness 14]. We also like to add a cautious caveat that our system might indeed be a granular system due to the strong morphology fluctuation at the surface.

In conclusion, we have observed the superconductorinsulator transition along with the evolution of the MR peak in amorphous InO films by varying their carrier density. A transition from a negative MR to a strong MR peak followed by the suppression of the MR peak was observed near the SIT by adding carriers. Strong MR peaks when the systems are insulating support the presence of localized Cooper pairs in the insulating regime and are qualitatively consistent with models based on there be- 
ing an effective superconducting granularity. Comparing the data with a theoretical simulation we argue that larger carrier density increases the paring energy $\Delta$, and therefore is responsible for both the SIT and the monotonic increase of the MR peak position to higher values of $H$. The EDLT configuration opens up the possibility of carrier-driven SIT studies of many other disordered systems.

We would like to thank Zvi Ovadyahu, Andrei Varlamov, Nandini Trivedi, Joe Mitchell, and Anirban Gangopadhyay for fruitful discussions and advice. This work was supported by the NSF under Grant No. NSF/DMR0854752 , by the NSF through the University of Minnesota MRSEC under Grant No. NSF/DMR-0819885, and by the US-Israel Binational Science Foundation under Grant No. 208299. Part of this work was carried out at the University of Minnesota Characterization Facility, a member of the NSF-funded Materials Research Facilities Network via the MRSEC program, and the Nanofabrication Center which receives partial support from the NSF through the NNIN program.

[1] A. M. Goldman and Nina Marković, Physics Today, Nov. 1998 p.39.

[2] Myles Steiner and Aharon Kapitulnik, Physica C 422, 16 (2005).

[3] Matthew P.A. Fisher, Phys. Rev. Lett, 65, 923 (1990).

[4] Yoseph Imry, and Zvi Ovadyahu, Phys. Rev. Lett. 49, 841 (1982).

[5] Amit Ghosal, Mohit Randeria, and Nandini Trivedi, Phys. Rev. B 65, 014501 (2001).

[6] Yonatan Dubi, Yigal Meir, and Yshai Avishai, Nature Letter, 449, 876 (2007).

[7] D. B. Haviland, Y. Liu, and A. M. Goldman, Phys. Rev. Lett. 62, 2180 (1989).

[8] T. I. Baturina et al., Phys. Rev. Lett. 99, 257003 (2007).

[9] D. Shahar and Z. Ovadyahu, Phys. Rev. B 46, 10917 (1992).

[10] G. Sambandamurthy, L.W. Engel, A. Johansson, and D. Shahar, Phys. Rev. Lett. 92, 107005 (2004).

[11] M. A. Paalanen, A. F. Hebard, and R. R. Ruel, Phys. Rev. Lett. 69, 1604 (1992).

[12] V. F. Gantmakher et al., JETP, 82, 951 (1996).

[13] I. Shammass et al., Phys. Rev. B 85, 140507 (2012).

[14] B. Sacépé et al., Nature Phys. 7, 239 (2011). also see the supplementary information.

[15] D. Sherman, G. Kopnov, D. Shahar, and A. Frydman, Phys. Rev. Lett. 108, 177006 (2012).

[16] Z. Ovadyahu, J. Phsy. C: Solid State Phys. 195187 (1986).

[17] Kevin A. Parendo et al., Phys. Rev. Lett. 94, 197004 (2005).

[18] Yeonbae Lee et al., Phys. Rev. Lett. 106, 136809 (2011).

[19] R. Misra, M. McCarthy, and A. F. Hebard, Appl. Phys. Lett. 90, 052905 (2007).

[20] D. Daghero et al., Phys. Rev. Lett. 108, 066807 (2012).

[21] A. L. Efros and B. I. Shklovskii, J. Phys. C: Solid State
Phys. 8, L49 (1975).

[22] A. Gerber et al., Phys. Rev. Lett. 78, 4277 (1997).

[23] A. I. Larkin and A. A. Varlamov, Theory of Fluctuations in Superconductors, OUP, Second Edition (2009).

[24] A. Glatz, A. A. Varlamov, and V. M. Vinokur, Phys. Rev. B 84, 104510 (2011).

[25] R. S. Thompson. Phys. Rev. B 1, 327 (1970).

[26] T. Kawaguti and Y. Shibuya, Phys. Lett. 45A, 395 (1973).

[27] B. L. Altshuler and A. G. Aronov, Solid State Comm. 46, 429 (1983).

[28] A. Johansson et al., Phys. Rev. Lett. 95, 116805 (2005).

[29] Y. Dubi, Y. Meir, and Y. Avishai, Phys. Rev. B 73, 054509 (2006).

[30] G. J. Conduit, Y. Meir, ArXiv 1111.2941 (2011).

[31] J. Mitchell, A. Gangopadhyay, V. Galitski, and M. Múller, Phys. Rev. B 85, 195141 (2012).

[32] Tianran Chen, Brian Skinner, and B. I. Shklovskii, Phys. Rev. B 86, 045135 (2012).

[33] A. V. Lopatin, and V. M. Vinokur, Phys. Rev. B 75, 092201 (2007).

[34] H. Q. Nguyen et al., Phys. Rev. Lett. 103, 157001 (2009).

[35] S. M. Hollen, et al., ArXiv 1301.6155 (2013).

[36] S. M. Hollen, et al., Phys. Rev. B 84, 064528 (2011).

[37] U. Givan and Z. Ovadyahu, Phys. Rev. B 86, 165101 (2012). 